\section{Elektrolytisch erzeugtes Berylliumäthyl-Radikal, stabilisiert mit Pyridin}

W. Strohmeier und G. Popp

Institut für Physikalische Chemie der Universität Würzburg

(Z. Naturforschg. 22 b, 891 [1967] ; eingegangen am 9. Mai 1967)

Im Zuge unserer Untersuchungen über die elektrolytische Abscheidung von Beryllium aus Berylliumorganischen Verbindungen bzw. Komplexverbindungen $^{1}$ wurde beobachtet, daß bei der Elektrolyse von Berylliumdiäthyl in Pyridin im Kathodenraum eine tiefblaue Lösung entsteht, welche ein scharfes ESRSignal mit einer Linenbreite von $4 \mathrm{Gauß}$ ergab. Der g-Faktor lag bei 2,004. Da frühere Messungen über die elektrolytische Leitfähigkeit metallorganischer Verbindung $\mathrm{MR}_{2}$ in Donatorlösungsmitteln zeigten, daß die Bruttogleichung der Ionenbildung durch Gl. (1) wiedergegeben werden kann, wobei das Kation $\mathrm{MR}^{\oplus}$

$$
\mathrm{MR}_{2} \rightleftarrows \mathrm{R}^{\ominus}+\mathrm{MR}^{\oplus}
$$

durch das Donatorlösungsmittel stabilisiert wird, vermuteten wir eine Entladung des mit Pyridin (Py) solvatisierten Kations $\mathrm{Be}\left(\mathrm{C}_{2} \mathrm{H}_{5}\right)^{\oplus}$ zu dem Radikal

1 W. Strohmeier u. F. Gernert, Z. Naturforschg. 20 b, 829 [1965].
$\mathrm{Be}\left(\mathrm{C}_{2} \mathrm{H}_{5}\right)$, das durch Pyridin unter Bildung von $\mathrm{Py}: \mathrm{Be}\left(\mathrm{C}_{2} \mathrm{H}_{5}\right) \cdot$ stabilisiert wird.

Führt man die Elektrolyse einer Lösung von Pyridin und Berylliumdiäthyl im Molverhältnis $1: 1$ über längere Zeit bei $60^{\circ}$ in einem U-Rohr mit Glasfritte zwischen zwei Kupfer-Elektroden durch, so bildet sich im Kathodenraum eine zähe schwarze Flüssigkeit, aus welcher sich nach Waschen mit Petroläther und anschließendem Trocknen im Hochvakuum eine hochviskose schwarze Substanz isolieren läßt, deren Analyse die Zusammensetzung $\mathrm{Py}: \mathrm{Be}\left(\mathrm{C}_{2} \mathrm{H}_{5}\right)$ ergab. $\mathrm{C}_{5} \mathrm{H}_{5} \mathrm{~N}: \mathrm{Be}\left(\mathrm{C}_{2} \mathrm{H}_{5}\right)$

$$
\begin{array}{llllll}
\text { Ber. } & \text { Be } 7,68 & \mathrm{NC}_{5} \mathrm{H}_{5} & 67,51 & \mathrm{C}_{2} \mathrm{H}_{5} & 24,75, \\
\text { Gef. } & \text { Be } 7,66 & \mathrm{NC}_{5} \mathrm{H}_{5} & 67,8 & \mathrm{C}_{2} \mathrm{H}_{5} & 23,6 .
\end{array}
$$

Die Substanz löst sich in Benzol. Die konzentrierte schwarze Lösung ergibt ein scharfes ESR-Signal mit einem g-Faktor von 2,004. Wird die Substanz jedoch in überschüssigem Pyridin (1:170) gelöst, so erhält man ein ESR-Signal mit Hyperfeinstruktur $(\sim 72 \mathrm{Li}$ nien). Da jedoch keine Liniengruppen auftraten, ist eine Zuordnung noch nicht möglich.

Der Deutschen Forschungsgemeinschaft und dem Fonds der Chemischen Industrie danken wir für die finanzielle Unterstützung dieser Arbeit.

2 W. Strohmeier u. F. Seifert, Z. Elektrochem. Ber. Bunsenges. physik. Chem. 60, 396 [1956].

\section{H-KMR-Spektren substituierter, paramagneti- scher Bis-cyclopentadienyl-metall-Komplexe}

\section{H. P. Fritz, H. J. Keller und K. E. Schwarzhans \\ Anorganisch-chemisches Laboratorium der Technischen Hochschule München \\ (Z. Naturforschg. 22 b, 891—892 [1967] ; eingegangen am 6. Mai 1967)}

In zwei ausführlichen Darstellungen haben wir über die Ergebnisse von ${ }^{1} \mathrm{H}-\mathrm{KMR}$-Untersuchungen an einigen paramagnetischen Bis-cyclopentadienyl-metall-Komplexen berichtet ${ }^{1,2}$, die vor allem durch die außergewöhnlichen Werte der ${ }^{1} \mathrm{H}-\mathrm{KMR}$-Kontaktverschiebungen dieser Verbindungsklasse ${ }^{3,4}$ angeregt wurden. Es konnte gezeigt werden, daß die Verschiebungen weder

1 H. P. Fritz, H. J. Keller u. K. E. Schwarzhans, J. organomet. Chem. 6, 652 [1966].

2 H. P. Fritz, H. J. Keller u. K. E. Schwarzhans, J. organomet. Chem. 7, 105 [1967].

3 H. M. McConnell u. C. H. Holm, J. chem. Physics 27, 314 [1957].

4 H. M. McConnell u. C. H. Holm, J. chem. Phyics 28, 749 [1958]. durch einen wesentlichen pseudo-Kontaktbeitrag noch durch den früher vorgeschlagenen Polarisationsmechanismus ${ }^{5}$ zu erklären sind. Um die Diskrepanz der beobachteten KMR-Linienbreiten zwischen kürzlich bekannt gewordenen Ergebnissen ${ }^{6}$ und unseren früheren Befunden aufzuklären ${ }^{7, *}$, haben wir vor alllem die Verbindungen Bis-(methyl-cyclopentadienyl)-kobalt (II) und -nickel(II) in der Schmelze eingehender KMRspektroskopisch bei verschiedenen Temperaturen untersucht, wobei die in Tab. 1 angeführten Werte erhalten wurden.

Für den Nickel-Komplex wurde sowohl im Bereich der Methyl- als auch der Cyclopentadienylprotonen oberhalb von $40{ }^{\circ} \mathrm{C}$ eine Linienaufspaltung beobachtet [Tab. 2]. Zudem tritt - in der Schmelze des reinen

5 D. A. Levy u. L. E. Orget, J. molecular Physics 3, 583 [1961].

6 M. F. Rettig u. R. S. Drago, Chem. Commun. 1966, 891.

7 H. P. Fritz, H. J. Keller u. K. E. Schwarzhans, Z. Naturforschg. 21 b, 809 [1966].

* In loc. cit. ${ }^{7}$ wurde versehentlich beim Druck das negative Vorzeichen weggelassen. In der ausführlichen Arbeit ${ }^{2}$ entsprechen die Vorzeichen den gefundenen Werten. 


\begin{tabular}{|c|c|c|c|c|c|}
\hline \multirow{2}{*}{$\begin{array}{l}\text { Temp. } \\
{\left[{ }^{\circ} \mathrm{C}\right]}\end{array}$} & \multicolumn{3}{|c|}{$\left(\mathrm{CH}_{3} \mathrm{C}_{5} \mathrm{H}_{4}\right)_{2} \mathrm{Co}^{\mathrm{a}}$} & \multicolumn{2}{|c|}{$\left(\mathrm{CH}_{3} \mathrm{C}_{5} \mathrm{H}_{4}\right)_{2} \mathrm{Ni} \mathrm{b}$} \\
\hline & $\mathrm{CH}_{3}$ & $\mathrm{~A}_{2}$ & $\mathrm{~B}_{2}$ & $\mathrm{CH}_{3}$ & Ring \\
\hline 30 & $-8,4$ & $+72,3$ & +49 & $-200,5 \mathrm{c}$ & +254 \\
\hline 40 & $-8,8$ & $+69,5$ & $+48,5$ & & \\
\hline 70 & $-9,6$ & $+63,8$ & $+45,7$ & & \\
\hline 85 & $-9,9$ & $+61,2$ & $+44,1$ & & \\
\hline
\end{tabular}

Tab. 1. Protonenresonanz-Signale von Bis (methylcyclopentadienyl)-kobalt(II) und -nickel(II) in Benzollösung [in ppm. bez. auf das Signal des $\left(\mathrm{C}_{5} \mathrm{H}_{5}\right)_{2} \mathrm{Fe}$. a Fehlergrenze: $\pm 0,1$ ppm. b Fehlergrenze: $\pm 3 \mathrm{ppm}$. c Linienbreite: $\sim 200 \mathrm{~Hz}$. d Linienbreite: $\sim 650 \mathrm{~Hz}$.

\begin{tabular}{ccccccccc}
\hline Temp. $\left[{ }^{\circ} \mathrm{C}\right]$ & 30 & 40 & 50 & 60 & 70 & 80 & 90 & 100 \\
\hline$[\mathrm{ppm}]^{*}$ & 458 & 439 & 429 & 421 & 405 & 396 & 385 & 376 \\
& - & 431 & 421 & 413 & - & - & - & -
\end{tabular}

Tab. 2. Protonenresonanz-Signale von Bis (methylcyclopentadienyl)-nickel(II) in der Schmelze [Abstand zwischen jeweils entsprechenden Methyl- und Ringprotonensignalen]. * Fehlergrenze: $\pm 3 \mathrm{ppm}$.

Nickelkomplexes - eine erhebliche Verbreiterung aller Absorptionen auf. Dieser Effekt erklärt, wie an der Variation der Linienenbreite von $18^{\circ}-40^{\circ} \mathrm{C}$ eindeutig gezeigt werden kann, auch die früher von uns angegebenen Werte ${ }^{2,7}$. Aufspaltung und Linienverbreiterung dürften entweder durch eine Dimerisierung oder eine intramolekulare Umlagerung hervorgerufen werden. Auf alle Fälle deutet die Verbreiterung auf eine Erhöhung der Elektronenspin-Relaxationszeit bzw. der Korrelationszeit in der Lösung hin. Mit Sicherheit kann eine Linienverbreiterung durch die Anwesenheit teilweise oxydierter Produkte ausgeschlossen werden, wie die Spektren von Proben nach verschieden langer Einwirkung von Luftsauerstoff eindeutig beweisen.

Wie bereits früher erwähnt ${ }^{7,8}$, kommt in den ${ }^{1} \mathrm{H}$ KMR-Spektren des Bis (methyl-cyclopentadienyl) -kobalt (II) der starke elektronische und sterische Einfluß der Methylsubstituenten auf die Anordnung der Liganden zum Ausdruck. Es wird eine Verzerrung des ursprünglich axial-symmetrischen Systems (vermutlich eine RingMetall-Ring-Abwinkelung sowie zusätzlich eine Abwinkelung in jedem Ringliganden um die $\mathrm{C}_{2}-\mathrm{C}_{5}$-Linie)

8 H. J. Keller u. H. Wawersik, J. organomet. Chem. 8, 185 [1967].

9 H. WAWERsik, Dissertation, Technische Hochschule, München 1967.

* Anm. b. d. Korr.: Bei $\left(\mathrm{CH}_{3}-\mathrm{C}_{5} \mathrm{H}_{4}\right)_{2} \mathrm{Cr}$ liegen ähnliche Verhältnisse vor. postuliert*. Auch in mehreren Rhodium-cyclopentadienyl-Komplexen läßt sich an Hand der IR-Spektren eine Symmetrieerniedrigung erkennen ${ }^{9}$.

Ein zusätzlicher Hinweis für diese Verzerrung ist aus dem UV-Spektrum des obigen Komplexes zu entnehmen. Man beobachtet im Gegensatz zu dem Nickelderivat eine Absorption bei $272 \mathrm{~m} \mu$, d. h. in einem Bereich, der für $\sigma$-gebundene Cyclopentadienyl-Liganden beschrieben wurde ${ }^{10}$.

Die ungewöhnlichen Verschiebungen der Protonensignale der Bis (cyclopentadienyl)-metall-Komplexe resultieren aus einer starken Wechselwirkung zwischen ungepaarten Elektronen in praktisch nicht-bindenden $\left[\left(\mathrm{a}_{1 \mathrm{~g}}+\mathrm{e}_{2 \mathrm{~g}}\right)\right.$ bzw. $\left.\left(\sigma_{\mathrm{g}}+\delta_{\mathrm{g}}\right)\right]$ Metallbahnfunktionen mit benachbarten Ringprotonen. Auf diese Weise wird ungepaarte Spindichte vom Zentralmetall direkt auf die Ringprotonen übertragen, so daß bei deren Resonanzsignalen wie z. B. im V $\left(\mathrm{C}_{5} \mathrm{H}_{5}\right)_{2}$ und $\mathrm{Cr}\left(\mathrm{C}_{5} \mathrm{H}_{5}\right)_{2}$ eine Verschiebung nach tieferen Feldern resultiert. Diese Modellvorstellung, die fast einer WasserstoffbrückenBindung zwischen Ring und Metall entspricht und durch spektroskopische wie präparative Befunde (niedere $\mathrm{C}-\mathrm{H}$-Valenzfrequenzen bei manchen Komplexen oder leichte Substituierbarkeit der Ringprotonen bei elektrophilem Angriff) gestützt wird, sagt für $\mathrm{Co}\left(\mathrm{C}_{5} \mathrm{H}_{5}\right)_{2}$ und $\mathrm{Ni}\left(\mathrm{C}_{5} \mathrm{H}_{5}\right)_{2}$,normales“ Verhalten eines aromatischen Radikals voraus. In diesen Komplexen bilden die praktisch nichtbindenden, nahezu bahnentarteten Metallbahnfunktionen eine voll besetzte Unterschale mit 6 Elektronen aus, so daß nur die in Molekülbahnfunktionen mit starkem Ligandenanteil untergebrachten ungepaarten Elektronen wirksam werden und eine Verschiebung der Signale hervorrufen.

Hier ergibt sich unter der Verwendung der bekannten Gleichungen die Spindichte an Wasserstoff- und Kohlenstoffatomen des Rings tatsächlich aus der Delokalisierung der ungepaarten Elektronen über die Liganden. Wie Messungen an einigen paramagnetischen Pyridinkomplexen gezeigt haben ${ }^{11}$, spielt die direkte Ubertragung freier Spindichte vom Metallion zu den magnetisch aktiven Protonen in allen Metallkomplexen, in denen Protonen sterisch günstig zum Metallion angeordnet sind, eine wesentliche Rolle, so daß in diesen Fällen eine Berechnung der Spindichte nach den bekannten Gleichungen keine sinnvollen Resultate liefert.

10 G. Wilkinson u. T. S. Piper, J. inorg. nuclear Chem. 2, 32 [1956].

11 H. P. Fritz, H. J. Keller u. K. E. Schwarzhans, noch unveröffentlichte Ergebnisse. 